\section{An elementary statistical package for 6502-based microcomputers}

\section{MICHAEL DAVID COOVERT \\ Ilinois State University, Normal, Ilinois 61761}

A software package written in BASIC for 6502 microprocessor-based microcomputers has been developed to perform the following elementary statistics: (1) descriptive statistics, including number of items, sum, arithmetic mean, geometric mean, harmonic mean, variance, standard deviation, median, range, and most probable error; (2) correlation analysis; (3) independentgroup $t$ test; (4) related-group t test; (5) simple chi square (with Yates correction if needed) and phi; (6) complex chi square and contingency coefficient; (7) frequency chi square; (8) F statistic; (9) one-way analysis of variance.
The programs are interactive, specifying the input format and requesting items by group and number. Suggestions are also provided for modifications to input data from files. The package can also be used in teaching elementary statistics via computer or simply for data analysis.

Limitations. The package was written in BASIC and developed for use on Ohio Scientific Challenger and APPLE microcomputers, but it should run with little or no modification on other 6502 -based systems, such as the KIM, SYM, AIM, and Commodore PET. Each program will reside and run in less than $4 \mathrm{~K}$ of memory.

Availability. Program listings are available at no charge from the author, Department of Psychology, Illinois State University, Normal, Illinois 61761.

(Accepted for publication September 14, 1980.) 